Images du travail, travail des images

$8 \mid 2020$

Filmer, travailler, chercher

\title{
Une maison au bord du monde. Un film de Pascal Cesaro, 74mn. Productions du Lagon, 2018
}

Jean-Paul Géhin

\section{OpenEdition}

Journals

Édition électronique

URL : http://journals.openedition.org/itti/352

DOI : $10.4000 /$ itti.352

Éditeur

Université de Poitiers

Référence électronique

Jean-Paul Géhin, « Une maison au bord du monde. Un film de Pascal Cesaro, 74mn. Productions du

Lagon, 2018 », Images du travail, travail des images [En ligne], 8 | 2020, mis en ligne le 01 février 2020, consulté le 14 avril 2021. URL : http://journals.openedition.org/itti/352 ; DOI : https://doi.org/10.4000/ itti.352

Ce document a été généré automatiquement le 14 avril 2021

Images du travail, travail des images 


\section{Une maison au bord du monde. Un film de Pascal Cesaro, 74mn. Productions du Lagon, 2018}

Jean-Paul Géhin

\section{RÉFÉRENCE}

Pascal Cesaro, Une Maison au bord du monde, Les Productions du Lagon, 2018, 74mn

Ce documentaire de recherche porte sur l'histoire de La maison, un centre de soins palliatifs créé en 1994 à Gardanne dans les Bouches du Rhône, par quelques soignants qui ont décidé de réagir face à l'épidémie du sida, en concevant un lieu spécialisé dans l'accompagnement de la fin de la vie. Malgré bien des difficultés liées aux peurs induites à l'époque par l'épidémie du Sida (ainsi des riverains se sont opposés au projet) et certaines résistances du corps médical aux soins palliatifs et au traitement de la douleur, La Maison a résisté et s'est développée. C'est aujourd'hui un établissement de Santé comprenant plusieurs unités, financé par l'état et reconnu d'utilité publique depuis 2011. 


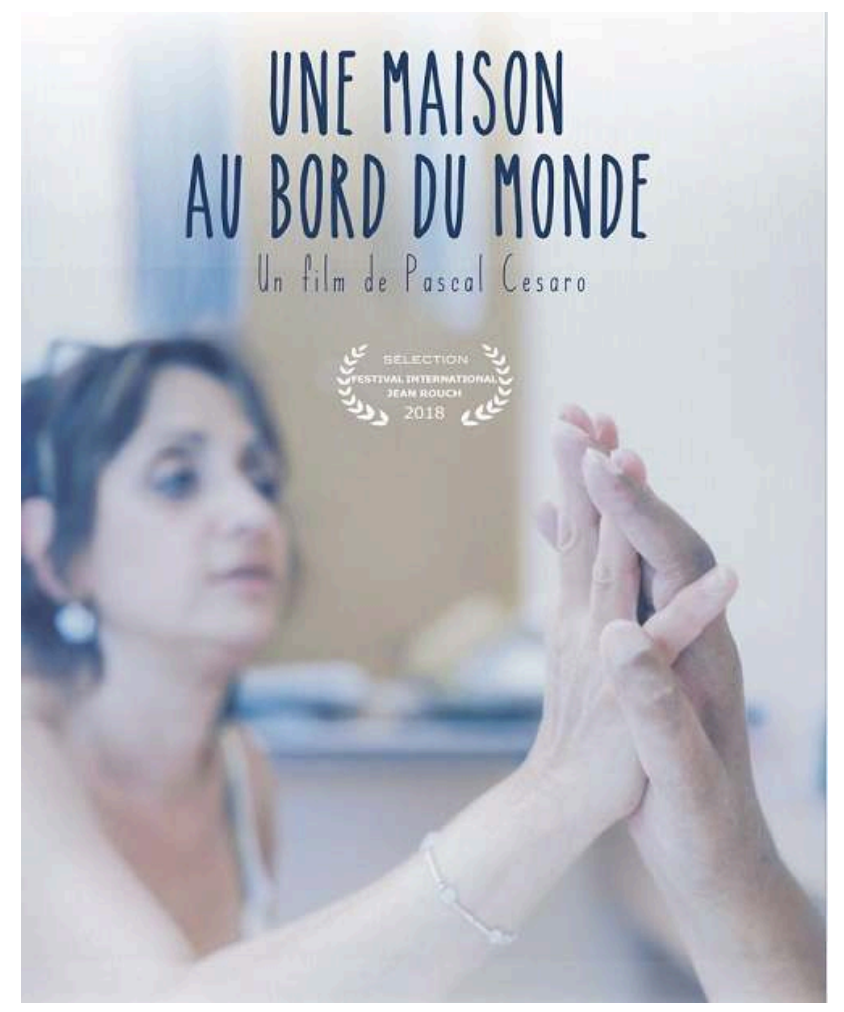

Les Productions du Lagon

2 Pascal Cesaro aborde la question du travail en soins palliatifs et de l'évolution du groupe professionnel, à l'occasion du départ à la retraite des fondateurs et donc du passage de relais entre deux générations de soignants dont les pratiques de soins sont marquées par la forte présence de la mort. Comment s'organise la relève? Quelles pratiques, quelles expériences et quelles valeurs les fondateurs souhaitent-ils transmettre? Quelles sont les spécificités du travail de soignants en soins palliatifs? Et aussi, et c'est une interrogation centrale pour le réalisateur/chercheur, comment montrer et mieux comprendre ce travail si particulier, impliquant un fort engagement?

3 Au fur et à mesure des pots de retraite et des échanges entre filmeur et filmés, quelques éléments de réponse se dessinent. D'abord, l'idée qui fait consensus que les changements touchent plutôt les patients que l'équipe : évolution des maladies, des publics, des traitements "qui rallongent la vie mais pas toujours en l'améliorant "; évolution aussi de l'institution qui, en étant de plus en plus reconnue, a considérablement grossi, rendant plus difficile l'ambiance familiale de la maison qui reste, pour tous les acteurs, essentielle; il s'agit de se distinguer des unités de soins palliatifs hospitaliers et de «se rapprocher du domicile, dans le décor, dans le fonctionnement ».

4 En revanche, concernant le travail en soins palliatifs, il y a accord pour souligner la permanence de l'engagement, l'investissement bien au delà de l'espace professionnel, l'importance de l'accompagnement des patients et des familles et un rapport au temps très particulier, comme le note le directeur historique : «On essaye de prendre le temps et, en même temps, le temps est compté (...) Chaque jour c'est un jour de vie en plus et c'est un jour à vivre en moins. » L'accent est mis aussi sur les choix très difficiles, 
notamment la question de la sédation et des rapports à la mort de l'équipe soignante et des proches, ce qui nécessite des procédures de supervision comme le rappelle une des personnages du film : «Je ne pense pas que je sois en souffrance mais, à des moments donnés, je suis triste.»

Pascal Cesaro connaît bien et de longue date La maison. Réalisateur de films de commande et de formation, il s'engage dans une thèse intitulée Le documentaire comme mode de production d'une connaissance partagée: recherche et développement d'une stratégie de réalisation, dont le terrain principal est l'équipe de soins palliatifs de Gardanne. Depuis la fin des années 1990, il a réalisé de nombreux documentaires sur La maison, dont trois directement dans le cadre de la thèse (Fragment $n^{\circ} 1$, L'annonciation à la Maison, LESA, La Maison, 75 mn, 2002 ; Fragment $n^{\circ} 2$ et $n^{\circ}$ 3, Instants de vie, LESA, La Maison, 62 $\mathrm{mn}$ et $75 \mathrm{mn}, 2003$ ), un film pour la télévision (Le jour d'avant. Chronique d'une unité de soins palliatifs, 13 Production, France3 Méditerranée, $52 \mathrm{mn}$, 2005) un court à la demande de l'équipe thérapeutique (Une journée dans la vie de Georges, La Maison, AMU, $26 \mathrm{mn}, 2007$ ) et enfin ce long métrage documentaire, qui a été sélectionné au festival international Jean Rouch et vient d'être édité en DVD (Une Maison au bord du monde, Productions du Lagon, CNRS audiovisuel, $74 \mathrm{mn}, 2018$ ). Ce travail documentaire dans le temps est un atout, qui lui permet non seulement de puiser dans les films précédents des séquences d'archives pour son dernier film mais aussi d'assumer une place importante dans le film. Le choix d'utiliser sa propre voix dans un commentaire off très présent, apparait judicieux car cela l'autorise à raconter simultanément l'histoire de La maison et celle de la relation filmeur/filmé et ce faisant de présenter la démarche de recherche.

L'auteur expérimente ainsi une démarche originale de recherche en sciences sociales mobilisant le film documentaire, conçu comme une co-construction filmeur/filmé ; ce qui permet dans ce cas de mieux comprendre les savoirs professionnels des soignants en soins palliatifs. Le terrain s'y prête car la parole y est très importante, la pensée et surtout la circulation de la pensée facilitant le travail collectif. Pour ce faire, s'inspirant des travaux de Douglas Harper, d'Antony M. Schwartz et de Yves Clot, il met en place un dispositif d'élicitation permettant de confronter les filmés à leur image et soulignant leur capacité de faire circuler les représentations et de construire ainsi des regards collectifs. 
Image 2

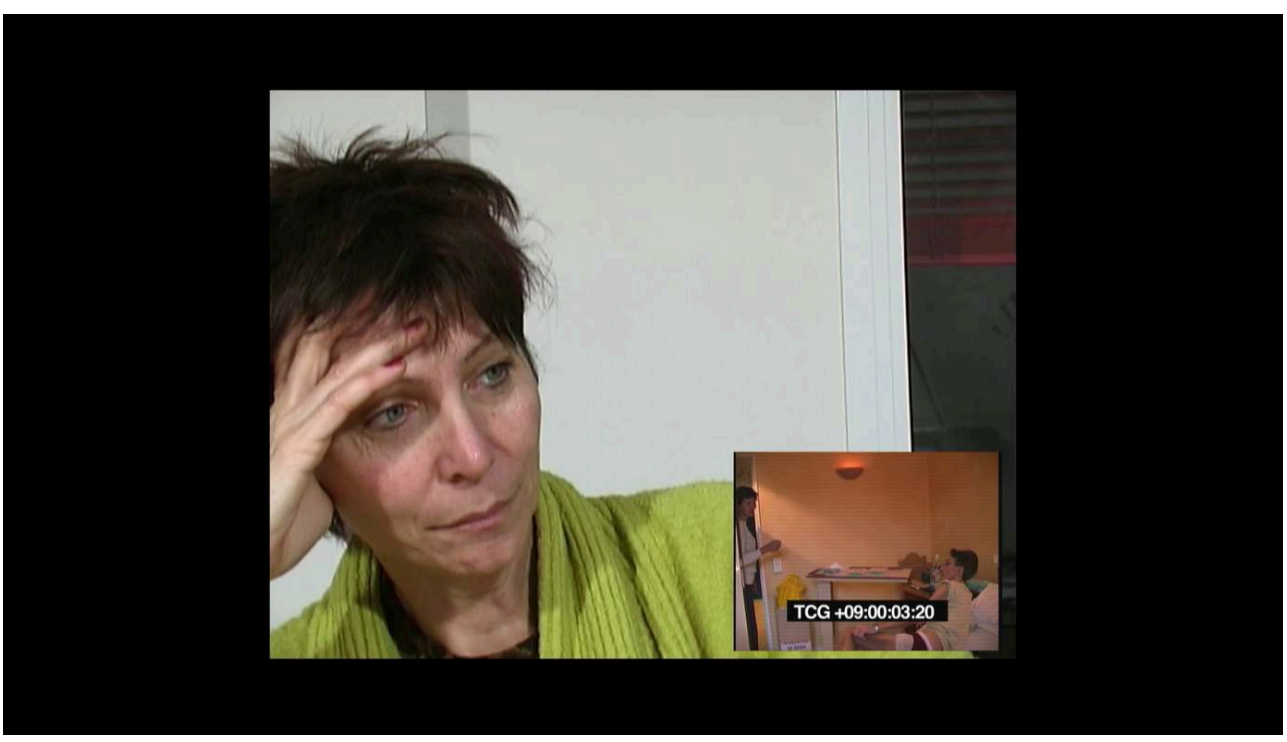

7 Une partie du dernier documentaire est construite autour d'un dispositif qui consiste à montrer à un filmé, qui apparaît dans un coin de l'écran, des séquences filmées de ses activités présentes ou passées. La voix off explicite la démarche : «J'élabore ce dispositif en miroir avec l'idée de montrer ce que je filme de leur travail pour voir comment ils construisent une réflexion sur leur activité (...) je saisis la difficulté pour eux d'établir une bonne distance dans la relation de soin et c'est dans ces moments là où j'apprends à filmer. Il me semble que face à mes images, ils s'interrogent sur leurs pratiques et ils deviennent co-auteurs d'une réflexion sur cette approche nouvelle des soins palliatifs. »

8 On aborde là un des aspects les plus intéressants du travail de réalisateur/chercheur de Pascal Cesaro. Engager une démarche réflexive qui laisse voir le travail de coconstruction des savoirs. Point d'autant plus notable que filmer les activités des professionnels des soins palliatifs, c'est bien souvent montrer les interactions soignants/patients sans montrer l'un des inter-actants, la pudeur et le respect des patients conduisant souvent la caméra à être face aux soignants, pour éviter tout risque de voyeurisme. Sur ce point, même si le dernier documentaire s'intéresse explicitement au travail des soignants, on peut regretter la quasi absence de scènes présentant les interactions soignants/soignés, sauf celles issues des archives des films précédents; ce qui s'explique sans doute par les conditions matérielles de production, plus proches des normes professionnelles dans le dernier documentaire: équipe de tournage plus conséquente alors que les images précédentes étaient du fait du seul chercheur; durée du temps passé sur le terrain beaucoup plus courte.

9 En ce qui concerne le bonus de $30 \mathrm{mn}$ figurant dans le DVD et présentant toute une série de spectacles réalisés dans le cadre de la promotion et sans doute du financement de la maison de Gardanne, on peut émettre un petit regret. Les spectacles ne sont pas du tout intégrés au documentaire alors qu'ils nous informent sur le travail en soins palliatifs, vécu comme vocation et nécessitant engagement et fort investissement. Ils soulignent la perméabilité des frontières entre travail et hors travail, et montrent la notoriété sur l'agglomération marseillaise du projet dans lequel sont impliqués soignants, patients, familles, artistes et bénévoles. Ces dimensions seront peut-être abordées dans un chapitre ultérieur de cette série documentaire. 


\section{BIBLIOGRAPHIE}

\section{Liste des productions audiovisuelles avec La Maison de Gardanne}

« Une Maison au bord du monde », Productions du Lagon, CNRS audiovisuel, 75mn, 2018

«Une journée dans la vie de Georges », La Maison, AMU, 26mn, 2007

"Le jour d'avant. Chronique d'une unité de soins palliatifs », 13 Production, France3 Méditerranée, 52 $\mathrm{mn}, 2005$

« Fragment $n^{\circ} 2$ et $n^{\circ}$ 3, Instants de vie », LESA, La Maison, $62 \mathrm{mn}$ et 75mn, 2003

« Fragment $n^{\circ}$ 1, L'annonciation à la Maison », LESA, La Maison, 75 mn, 2002

\section{AUTEURS}

\section{JEAN-PAUL GÉHIN}

Jean-Paul Géhin est enseignant chercheur à l'Université de Poitiers et membre du GRESCO. Il a publié de nombreux articles et ouvrages en sociologie du travail et plus particulièrement sur la question des modalités d'articulation entre éducation et travail. Responsable et enseignant dans des masters de réalisation documentaire, il s'intéresse à la médiation scientifique et à l'usage des images en sciences sociales. Dans ce cadre, il a participé à la fondation en 2009 du festival Filmer le travail qu'il préside actuellement. Il fait également partie de la direction collégiale de la nouvelle revue numérique et pluridisciplinaire Images du travail, travail des images. 